Supplement of Nat. Hazards Earth Syst. Sci., 19, 269-285, 2019

https://doi.org/10.5194/nhess-19-269-2019-supplement

(c) Author(s) 2019. This work is distributed under

the Creative Commons Attribution 4.0 License.

(c) (1)

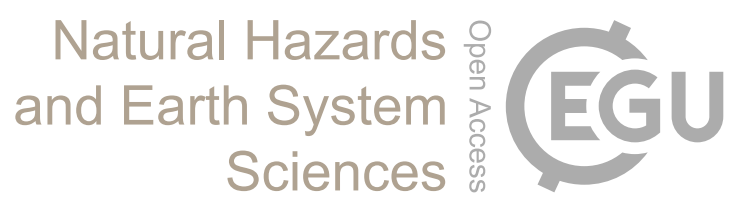

Supplement of

\title{
A method of deriving operation-specific ski run classes for avalanche risk management decisions in mechanized skiing
}

Reto Sterchi and Pascal Haegeli

Correspondence to: Reto Sterchi (reto_sterchi@sfu.ca)

The copyright of individual parts of the supplement might differ from the CC BY 4.0 License. 
Table S1: Qualitative run characterization with attribute names and levels.

\begin{tabular}{|c|c|c|}
\hline $\begin{array}{l}\text { Attribute } \\
\text { name }\end{array}$ & Question & Levels \\
\hline $\begin{array}{l}\text { Access } \\
\text { Required } \\
\text { flying } \\
\text { conditions } \\
\text { Particular } \\
\text { pickup } \\
\text { features }\end{array}$ & $\begin{array}{l}\text { How do you generally feel } \\
\text { about the accessibility of } \\
\text { this run when the cluster } \\
\text { of runs is accessible? } \\
\text { What other access feature } \\
\text { of the pickup(s) of this run } \\
\text { stand out? }\end{array}$ & $\begin{array}{l}\text { 1. I can always get to this run. } \\
\text { 2. It is often possible to make this work. } \\
\text { 3. Conditions need to line up to make this work. } \\
\text { 4. Flying conditions need to be perfect to consider this run } \\
\text { 1. Avalanche overhead hazard during regular cycles. } \\
\text { 2. Avalanche overhead hazard during large cycles. } \\
\text { 3. Common presence of triggers for overhead avalanche hazards }\end{array}$ \\
\hline $\begin{array}{l}\text { Type of Terrain } \\
\text { Type of } \\
\text { terrain }\end{array}$ & $\begin{array}{l}\text { What type(s) of skiing } \\
\text { terrain does this run } \\
\text { include? }\end{array}$ & $\begin{array}{l}\text { 1. Glaciated terrain } \\
\text { 2. Non-glaciated alpine terrain } \\
\text { 3. Extreme alpine terrain (faces) } \\
\text { 4. Open slopes at tree line or below tree line } \\
\text { 5. Glades at tree line or below tree line } \\
\text { 6. Open canopy/snow forest (individual tree crowns do not overlap) } \\
\text { 7. Burnt forest } \\
\text { 8. Cut blocks } \\
\text { 9. Large avalanche path formed from above } \\
\text { 10. Dense forest } \\
\text { 11. Open planar slopes } \\
\text { 12. Highly featured/convoluted terrain }\end{array}$ \\
\hline $\begin{array}{l}\text { Skiing Experien } \\
\text { Skiing } \\
\text { difficulty }\end{array}$ & $\begin{array}{l}\text { What is the difficulty level } \\
\text { of this run when } \\
\text { conditions are good? }\end{array}$ & $\begin{array}{l}\text { 1. Easy } \\
\text { 2. Moderate } \\
\text { 3. Challenging }\end{array}$ \\
\hline $\begin{array}{l}\text { Overall guest } \\
\text { experience }\end{array}$ & $\begin{array}{l}\text { When the conditions on } \\
\text { this run are good, what is } \\
\text { your opinion of the overall } \\
\text { skiing experience that his } \\
\text { run offers? }\end{array}$ & $\begin{array}{l}\text { 1. Poor (Happy to move on) } \\
\text { 2. Fair (Not bad skiing) } \\
\text { 3. Good (A good product) } \\
\text { 4. Very good (This is why guests come back for more) } \\
\text { 5. Exceptional (Life changing mountain experience) }\end{array}$ \\
\hline
\end{tabular}


Table S1: Continued.

\begin{tabular}{|c|c|c|}
\hline $\begin{array}{l}\text { Attribute } \\
\text { name }\end{array}$ & Question & Levels \\
\hline \multicolumn{3}{|c|}{ Operational Role(s) } \\
\hline $\begin{array}{l}\text { Operational } \\
\text { role(s) }\end{array}$ & $\begin{array}{l}\text { What particular } \\
\text { operational role(s) does } \\
\text { this run have in your } \\
\text { program? }\end{array}$ & $\begin{array}{l}\text { 1. Safe and accessible under almost all conditions run } \\
\text { 2. Signature run (defines your operation) } \\
\text { 3. Destination run (objective of a circuit) } \\
\text { 4. Bread and butter run (high efficiency production run) } \\
\text { 5. Key jump run (might not have good skiing, but makes a circuit work) } \\
\text { 6. Time management run (can be used to keep busy for a while, e.g., } \\
\text { during fuel run) } \\
\text { 7. Regular lunch run } \\
\text { 8. Not preferred run (only considered if running out of options for } \\
\text { reasonable skiing) } \\
\text { 9. Open season run (only considered under bombproof conditions) } \\
\text { 10. Rarely visited, but important under special circumstance }\end{array}$ \\
\hline $\begin{array}{l}\text { Hazard Potent } \\
\text { Steepness }\end{array}$ & $\begin{array}{l}\text { What is the steepness of } \\
\text { the most serious slopes on } \\
\text { this run? }\end{array}$ & $\begin{array}{l}\text { 1. Gentle (no significant avalanche slopes on ski lines) } \\
\text { 2. Moderately steep (concerned about avalanches under specific } \\
\text { condition) } \\
\text { 3. Moderate with steep pitches (always concerned about avalanches) } \\
\text { 4. Sustained steep (always concerned about avalanches) }\end{array}$ \\
\hline $\begin{array}{l}\text { Exposure to } \\
\text { avalanche } \\
\text { slopes on the } \\
\text { ski line(s) }\end{array}$ & $\begin{array}{l}\text { If moderately steep or } \\
\text { steep, what is the exposure } \\
\text { to avalanche slopes on this } \\
\text { run? }\end{array}$ & $\begin{array}{l}\text { 1. A single smaller avalanche slope capable of producing Size } 1.5-2.5 \\
\text { 2. Multiple smaller avalanche slopes capable of producing Size } 1.5-2.5 \\
\text { 3. Large avalanche slopes producing Size } 3.0 \text { or larger }\end{array}$ \\
\hline $\begin{array}{l}\text { Avalanche } \\
\text { related } \\
\text { terrain } \\
\text { hazards }\end{array}$ & $\begin{array}{l}\text { What avalanche related } \\
\text { terrain hazards stand out } \\
\text { on this run? }\end{array}$ & $\begin{array}{l}\text { 1. Avalanche overhead hazard during regular cycles (Size } 3.0 \text { or smaller) } \\
\text { 2. Avalanche overhead hazard during large events only (Size } 3.5 \text { or } \\
\text { larger) } \\
\text { 3. Common presence of triggers for overhead avalanche hazard (e.g., ice } \\
\text { fall, cornice) } \\
\text { 4. Unavoidable unsupported terrain shapes } \\
\text { 5. Lack of surface roughness } \\
\text { 6. Frequent performers that retain hazard and wait for human triggering } \\
\text { 7. Frequent natural avalanche which stabilize the slope } \\
\text { 8. High consequence terrain when caught }\end{array}$ \\
\hline $\begin{array}{l}\text { Other } \\
\text { hazards }\end{array}$ & $\begin{array}{l}\text { What other hazards stand } \\
\text { out on this run? }\end{array}$ & $\begin{array}{l}\text { 1. Crevasse hazard, isolated } \\
\text { 2. Crevasse hazard, widespread and/or unavoidable } \\
\text { 3. Cornices directly affecting the ski line(s) } \\
\text { 4. Tree well hazard } \\
\text { 5. Open creeks, vent holes, rock crevasses } \\
\text { 6. Particularly large tree bombs } \\
\text { 7. Potentially particularly challenging for rescues and/or finding a lost } \\
\text { skier }\end{array}$ \\
\hline $\begin{array}{l}\text { Overall } \\
\text { friendliness }\end{array}$ & $\begin{array}{l}\text { In terms of hazards, what } \\
\text { is your sense of the overall } \\
\text { friendliness of the terrain } \\
\text { on this run? }\end{array}$ & $\begin{array}{l}\text { 1. Very friendly } \\
\text { 2. Friendly } \\
\text { 3. Neutral } \\
\text { 4. Unfriendly } \\
\text { 5. Very unfriendly }\end{array}$ \\
\hline
\end{tabular}


Table S1: Continued.

\begin{tabular}{lll}
\hline $\begin{array}{l}\text { Attribute } \\
\text { name }\end{array}$ & Question & Levels \\
\hline $\begin{array}{lll}\text { Guide-ability } \\
\text { Guide-ability }\end{array}$ & $\begin{array}{l}\text { What is your opinion of } \\
\text { the guide-ability of this } \\
\text { run? }\end{array}$ & $\begin{array}{l}\text { 1. Very easy (i.e., the terrain naturally leads guests to the right line) } \\
\text { 2. Easy } \\
\text { 3. Difficult } \\
\text { 4. Very difficult (i.e., requires detailed instructions and a close eye on } \\
\text { the guest) }\end{array}$ \\
\hline
\end{tabular}


Table S2: Average seasonal and overall percentages of run list ratings for the six groups of similarly managed ski runs at NEH.

\begin{tabular}{|c|c|c|c|c|c|c|c|c|}
\hline Group & $\mathbf{n}$ & Run list rating & 2013 & 2014 & 2015 & 2016 & 2017 & Overall \\
\hline \multirow[t]{3}{*}{1} & 8 & open & $97 \%$ & $97 \%$ & $94 \%$ & $98 \%$ & $>99 \%$ & $97 \%$ \\
\hline & & closed due to avalanche hazard & $<1 \%$ & $3 \%$ & $<1 \%$ & $<1 \%$ & $<1 \%$ & $1 \%$ \\
\hline & & other hazards / not discussed & $2 \%$ & $0 \%$ & $5 \%$ & $1 \%$ & $0 \%$ & $2 \%$ \\
\hline \multirow[t]{3}{*}{2} & 9 & open & $95 \%$ & $79 \%$ & $61 \%$ & $91 \%$ & $>99 \%$ & $86 \%$ \\
\hline & & closed due to avalanche hazard & $1 \%$ & $21 \%$ & $3 \%$ & $5 \%$ & $<1 \%$ & $6 \%$ \\
\hline & & other hazards / not discussed & $4 \%$ & $0 \%$ & $36 \%$ & $4 \%$ & $0 \%$ & $9 \%$ \\
\hline \multirow[t]{3}{*}{3} & 2 & open & $98 \%$ & $90 \%$ & $69 \%$ & $97 \%$ & $63 \%$ & $84 \%$ \\
\hline & & closed due to avalanche hazard & $0 \%$ & $10 \%$ & $<1 \%$ & $0 \%$ & $1 \%$ & $2 \%$ \\
\hline & & other hazards / not discussed & $2 \%$ & $0 \%$ & $30 \%$ & $3 \%$ & $36 \%$ & $15 \%$ \\
\hline \multirow[t]{3}{*}{4} & 13 & open & $87 \%$ & $80 \%$ & $79 \%$ & $74 \%$ & $85 \%$ & $81 \%$ \\
\hline & & closed due to avalanche hazard & $10 \%$ & $20 \%$ & $5 \%$ & $14 \%$ & $12 \%$ & $12 \%$ \\
\hline & & other hazards / not discussed & $3 \%$ & $0 \%$ & $16 \%$ & $12 \%$ & $3 \%$ & $7 \%$ \\
\hline \multirow[t]{3}{*}{5} & 13 & open & $56 \%$ & $28 \%$ & $61 \%$ & $53 \%$ & $36 \%$ & $47 \%$ \\
\hline & & closed due to avalanche hazard & $42 \%$ & $71 \%$ & $30 \%$ & $39 \%$ & $64 \%$ & $49 \%$ \\
\hline & & other hazards / not discussed & $2 \%$ & $1 \%$ & $9 \%$ & $8 \%$ & $0 \%$ & $4 \%$ \\
\hline \multirow[t]{3}{*}{6} & 14 & open & $31 \%$ & $18 \%$ & $35 \%$ & $33 \%$ & $25 \%$ & $29 \%$ \\
\hline & & closed due to avalanche hazard & $67 \%$ & $82 \%$ & $36 \%$ & $50 \%$ & $70 \%$ & $61 \%$ \\
\hline & & other hazards / not discussed & $2 \%$ & $<1 \%$ & $29 \%$ & $17 \%$ & $5 \%$ & $10 \%$ \\
\hline
\end{tabular}




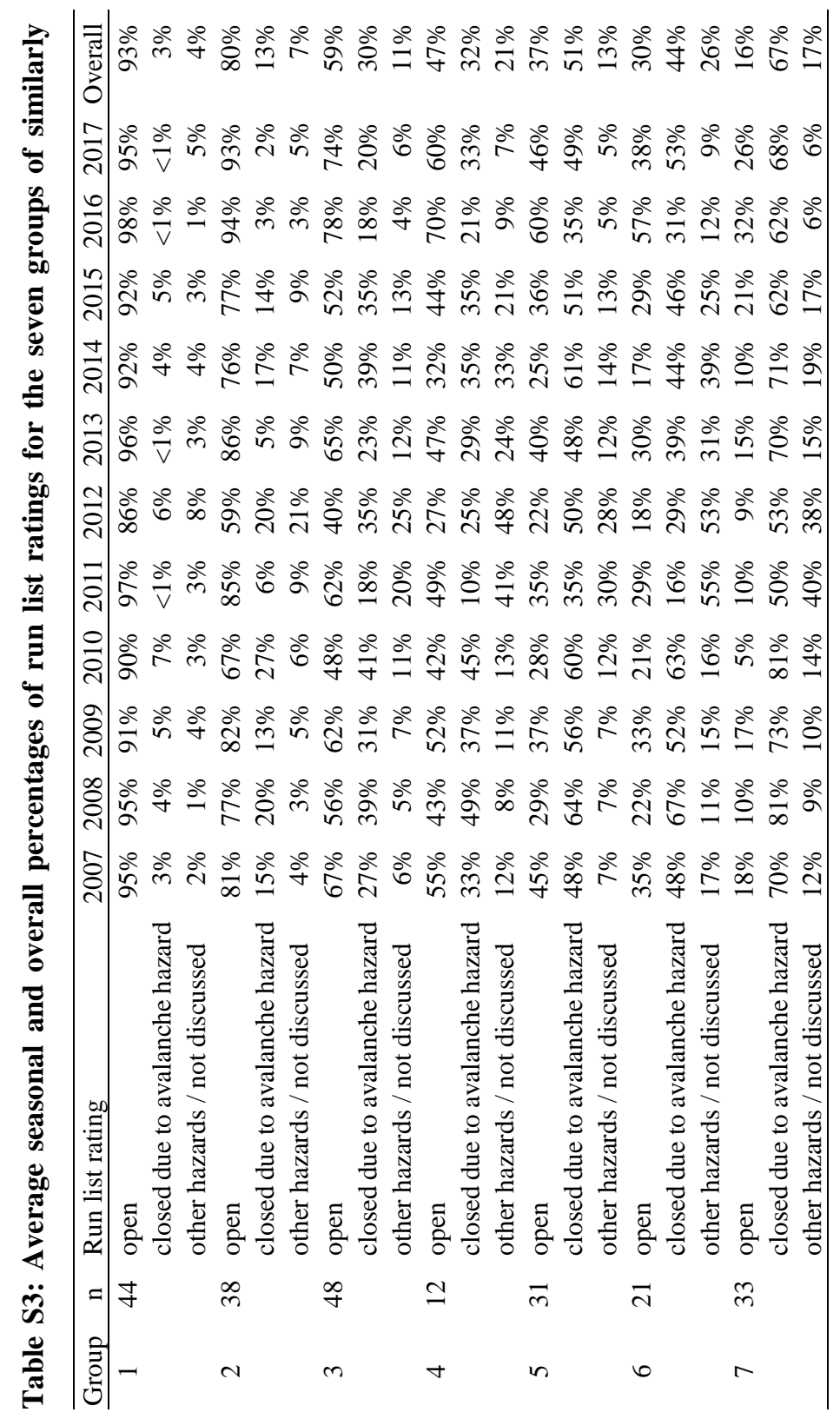

